\section{MS45-O4 Background modelling in the presence of ice rings}

James M. Parkhurst ${ }^{1,2}$, Andrea Thorn ${ }^{2}$, Graeme Winter ${ }^{1}$, David Waterman $^{3}$, Luis Fuentes-Montero ${ }^{1}$, Richard Gildea ${ }^{1}$, Garib Murshudov $^{2}$, Gwyndaf Evans ${ }^{1}$

\author{
1. Diamond Light Source \\ 2. LMB, Cambridge \\ 3. $\mathrm{CCP} 4$
}

email: james.parkhurst@diamond.ac.uk

In macromolecular crystallography, integration programs are used to estimate the intensities of individual Bragg reflections. In the simple case of "summation integration", this is typically done by labelling each pixel in the neighbourhood of a Bragg peak as either foreground or background; the reflection's intensity is then estimated as the total number of counts in the foreground region minus the total background estimated in the foreground region. The background in the foreground region is estimated from the surrounding background pixels.

Typically, the background is modelled as either a constant (Kabsch 2010) or a plane (Leslie 1999) in the region of the reflection peak. In most cases, these models are reasonably appropriate for the data. However, they may not be appropriate when there are additional features in the background, most notably ice rings; the shape of the background at the peak of an ice ring is not well modelled by a constant or plane.

156 Pilatus datasets were selected from the JCSG database. In total, 15 datasets were observed to have ice rings. The data were processed with DIALS (Waterman et al. 2013) and scaled with POINTLESS and AIMLESS (Evans and Murshudov 2013). Analysis of the distribution of intensities as a function of resolution revealed that, at ice ring resolutions, the reflection intensities were being systematically over-estimated. This indicates that the reflection backgrounds at ice ring resolutions were being systematically under-estimated.

We present a simple method, implemented in the DIALS framework, for modelling the background in the presence of ice rings. The method involves the creation of a global background model that describes the expected shape of the background at every pixel in the image. This method is shown to result in better background estimates (and consequently intensity estimates) for reflections recorded on ice rings than the simple constant or planar models.

Leslie, A. G. W. (1999). Acta Crystallogr. D. Biol. Crystallogr. 55, 1696-1702.

Evans, P. R. \& Murshudov, G. N. (2013). Acta Crystallogr. D. Biol. Crystallogr. 69, 1204-1214.

Kabsch, W. (2010). Acta Crystallogr. Sect. D Biol. Crystallogr. 66, 133-144.

Waterman, D. G. et al. (2013). CCP4 newsletter on protein crystallography, $49,16-19$

Keywords: Integration, Background, Ice Rings

\section{MS45-O5 At home accurate XRD measurements with an hybrid pixel X-ray detector: a comparison with $\mathrm{CCD}$, APS-CMOS commercial detectors and theoretical model}

Claude Lecomte ${ }^{1}$, Emmanuel Wenger ${ }^{1}$, Paul Alle ${ }^{1}$, Emmanuel Aubert ${ }^{1}$, Slimane Dahaoui ${ }^{1}$, Dominik Schaniel ${ }^{1}$

\section{Université de Lorraine}

email: claude.lecomte@univ-lorraine.fr

The new generation of X-ray detectors, the hybrid pixel area detectors or 'pixel detectors', are based on direct detection and single-photon counting processes. Large linearity range, high dynamic and extremely low noise leading to unprecedented high signal-to-noise ratio for bi-dimensional X-ray detectors, fast readout time (high frame rates) and electronic shutter are among their intrinsic characteristics which render them very attractive. As shown in our previous study on sodium nitroprusside crystals [Wenger et al. Acta Cryst B, 2014], it was demonstrated that these detectors are promising at laboratory sources for quasi-static experiments and accurate electron density measurements [Alle et al., Physica Scripta, 2016]. In this study, four different electron density models of a weakly scattering pure organic compound, the 4-benzyloxy-3-methoxybenzaldehyde $\left(\mathrm{C}_{15} \mathrm{H}_{14} \mathrm{O}_{3}\right)$ have been refined against experimental structure factors obtained from commercial CCD and CMOS type X-ray detectors, an XPAD hybrid pixel detector and theoretical structure factors from DFT calculations. Atom coordinates, thermal displacement parameters, critical points, electron density values, laplacien, valence populations and electrostatic potential will be discussed and compared.

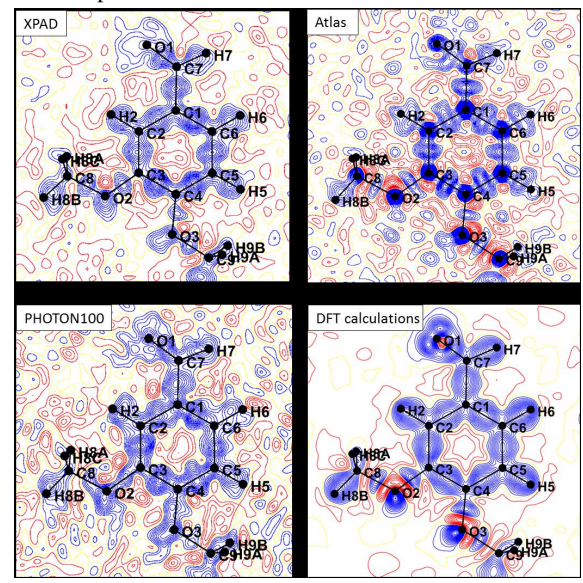

Figure 1. Electron density deformation maps in the C1-C6 plane from the three different detectors data sets and from DFT calculations. Max Res $=0.96 \mathrm{~A}^{-1}$ With all $\mathrm{I}>0$ reflections. Isocontours: $0.05 \mathrm{e} / \AA$

Keywords: X-ray detectors, hybrid pixel detector, CCD detectors, CMOS detectors, laboratory accurate X-ray intensity 
MS46 Computational tools for theoretical chemistry in crystallography

\section{MS46-01 Recent Advancements in the Development of X-ray Constrained Wave} Function Strategies

\author{
Alessandro Genoni ${ }^{1}$
}

1. CNRS \& Université de Lorraine, Laboratorie SRSMC - UMR 7565, Vandoeuvre-lès-Nancy, France

email: Alessandro.Genoni@univ-lorraine.fr

As well known, the wave function is a fundamental entity that intrinsically contains all the information of a system in the most compact way. For this reason the possibility of determining wave functions from experimental data has been a tantalizing perspective that motivated different research groups over the years.

Among the modern strategies proposed in this context, the X-ray constrained wave function (XC-WF) method introduced by Jayatilaka [1] is undoubtedly the most noteworthy. This technique can be considered as the most promising advancement of the pioneering strategies introduced by Clinton et al. [2] and it consists in extracting single Slater determinants that, other than minimizing the Hartree-Fock energy of the systems, reproduce sets of experimental structure factors within a predefined accuracy.

In our group, the XC-WF approach has been extended in order to extract Extremely Localized Molecular Orbitals (ELMOs) from experimental X-ray diffraction data [3-4], namely Molecular Orbitals that are strictly localized on small molecular fragments (e.g., atoms or bonds) and that are consequently very close to the traditional chemical picture of molecules. Determining $\mathrm{XC}$-ELMOs is straightforward and the new strategy can be seen as an alternative tool to determine experimental electron densities, combining the quantum mechanical rigor of the wave function-based approaches with the chemical interpretability of the popular multipole model.

More recently, always starting from the concept of ELMOs, we have also devised a preliminary X-ray constrained Valence Bond method. This technique, other than being the first attempt of introducing a multi-determinant wave function ansatz in the Jayatilaka approach, has allowed us to successfully study the charge distribution of the syn-1,6:8,13-Biscarbonyl[14]annulene at different pressures [5], theoretically confirming the partial rupture of the aromaticity experimentally observed when pressure is increased [6].

An overview of our techniques recently developed in the framework of the XC-WF approach will be presented. 